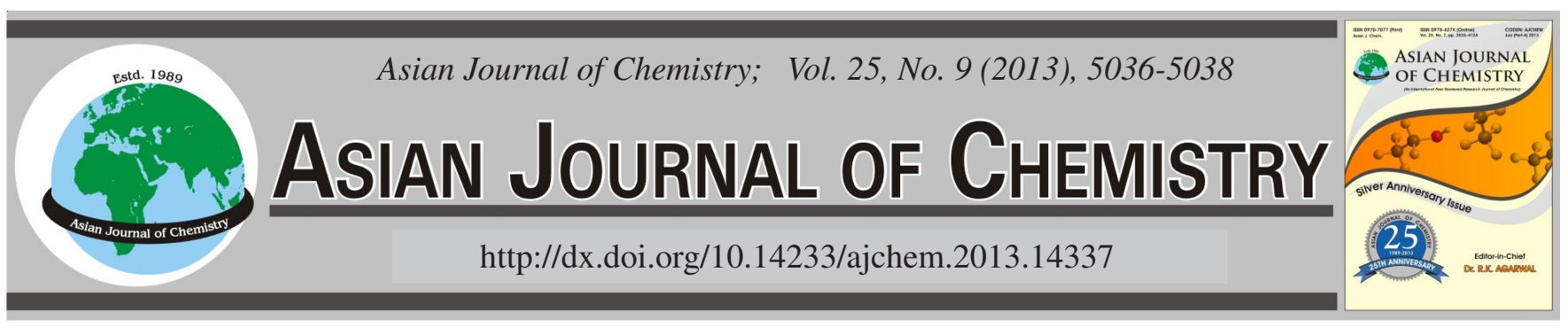

\title{
Polyaniline/Graphene-Based Electrochemical Biosensor for Sensitive Detection of Glucose
}

\author{
Yuqing Ge ${ }^{1}$, Zhihua Chen ${ }^{2}$, Qinghui Jin ${ }^{1, *}$, Hongu Mao ${ }^{1}$, JiAnshe Liu $^{2}$ and Jianlong Zhao ${ }^{1, *}$
}

${ }^{1}$ State Key Lab of Transducer Technology, Shanghai Institute of Microsystem and Information Technology, Chinese Academy of Science, Shanghai 200050, P.R. China

${ }^{2}$ School of Environmental Science and Engineering, Donghua University, Shanghai 201620, P.R. China

*Corresponding author: Fax: +86 21 62511070-8709; Tel: +86 21 62511070-8703; E-mail: jinqh@mail.sim.ac.cn; yq_ge@hotmail.com; jlzhao@mail.sim.ac.cn

\begin{abstract}
A novel approach for glucose biosensor fabrication was described. It based on immobilization of glucose oxidase on polyaniline/graphene composite membrane modified glassy carbon electrode. Cyclic voltammetry demonstrated that modified electrodes owned good electrochemical activity, superior sensitivity and high stability. The good electrocatalytical activity toward $\mathrm{H}_{2} \mathrm{O}_{2}$ and $\mathrm{O}_{2}$ might be attributed to the synergistic effect of graphene and polyaniline. The results showed that the electrochemical biosensor had a wide linear response from 0.1 to $10 \mathrm{mM}$.
\end{abstract}

Key Words: Graphene, Polyaniline, Glucose oxidase, Electrochemical biosensor.

\section{INTRODUCTION}

The electrochemical biosensors utilizing nanomaterials have recently attracted considerable attention in the area of sensing. Many kinds of nanomaterials, such as carbon nanotubes, gold nanoparticles, metal oxides and semiconductors, have been used in biosensors in the medicine and food quality control field $^{1-6}$. The introduction of nanomaterials with unique properties could improve the performance of biosensor, e.g., simplicity, speed, high selectivity and high sensitivity.

The emergence of graphene has recently opened up an exciting field in the science and technology. Graphene is a flat monolayer of carbon atoms tightly packed into a two-dimensional (2D) honeycomb lattice with extraordinary electronic transport properties and high electrocatalytic activities ${ }^{7-9}$. They have the potential applications as electrode materials in optoelectronic devices, super-capacitors, ultrasensitive chemical sensors and biosensors ${ }^{10-14}$. Moreover, graphene based eletrodes have shown superior performance in terms of electocatalytic than corbon nanotube ones ${ }^{15,16}$.

Despite their promising applications in the biomedical research, the development of electrochemical biosensors with improved sensitivity and low detection limit has remained a great challenge. Especially, because of the usefulness in diagnostic analysis of diabetes, glucose biosensors based on nanomaterials have been extensively studied. In this approach, we demonstrate a new approach to improve the sensitivity of glucose electrochemical biosensor by introducing graphene oxides and polyaniline (PANI) into its construction. Graphene oxides not only could act as an advanced support with very large surface area for immobilizing glucose oxidases (GOD $)^{17}$, but also effectively promote the electron transfer between electrode and analytes. Characterization of the interconnection between polyaniline and graphene oxides was performed using scanning electron microscope (SEM) analyses. Cyclic voltammetry $(\mathrm{CV})$ demonstrated that polyaniline was electroactive in the neutral after interconnected with graphene oxides and own well charge-transfer interaction. With glucose oxidases as an enzyme model, the electrochemical biosensor maintained its bioactivity with linear glucose response up to $0.1 \mathrm{mM}$.

\section{EXPERIMENTAL}

Graphene oxides were synthesized from graphite powder through a modified Hummers method ${ }^{18}$. As-purified graphene oxides suspensions were dispersed in water to prepare a 0.05 wt $\%$ dispersion by ultrasonication for $0.5 \mathrm{~h}$. Polyaniline were synthesized by the typical interfacial reaction ${ }^{19}$.

General procedure: Typically, the homogeneous graphene oxides aqueous suspension $5 \mathrm{~mL}$ was added to 1 $\mathrm{mL}$ polyaniline solution. The mixture was stirred for $2 \mathrm{~h}$ and then centrifuged. The supernatant were dropped on polished glassy carbon electrode (GCE, diameter $3 \mathrm{~mm}$ ). The modified electrodes were obtained after drying at the room temperature. Then after the removal of oxygen $20 \mathrm{~min}$ for $1 \mathrm{mg} / \mathrm{mL}$ GOD PBS buffer (0.01 M $o$-phenylenediamine), the modified 
electrode was electrochemical polymerized $0.5 \mathrm{~h}$ in a $0.65 \mathrm{~V}$ constant voltage. Then the GOD modified electrode was moved into a refrigerator and kept at $4{ }^{\circ} \mathrm{C}$ to dry overnight. During the experimental period, the modified eletrodes were stored at $4{ }^{\circ} \mathrm{C}$ until use.

Detection method: All electrochemical experiments were performed by a CHI660 electrochemical workstation with a three-electrode system. GCE served as the working electrode, a platinum wire as the auxiliary electrode and an $\mathrm{Ag} / \mathrm{AgCl} / 3.0$ $\mathrm{M} \mathrm{KCl}$ as the reference electrode, respectively. Cyclic voltammetric experiments were carried out in a quiescent solution at $50 \mathrm{mV} \mathrm{s}^{-1}$ in an electrochemical cell filled with $10 \mathrm{~mL}$ phosphate buffered saline (PBS) solution. The morphology of the samples was determined by SEM (FEI, Sirion-200).

\section{RESULTS AND DISCUSSION}

The PANI dispersed graphene were characterized with SEM. The SEM images in Fig. 1 exhibited surface morphology of graphene, PANI and PANI/graphene complex. Graphene was a thin layer with flexible edge, while there were some folds. The PANI morphology is an irregular tenuous, interconnected fibrillar network. The gaps between the sheets were not conducive to the overall conductivity of PANI. Graphene/ PANI complex were connected together to form a uniform sheet, which were benefit the enhancement of conductivity and electrochemical properties.
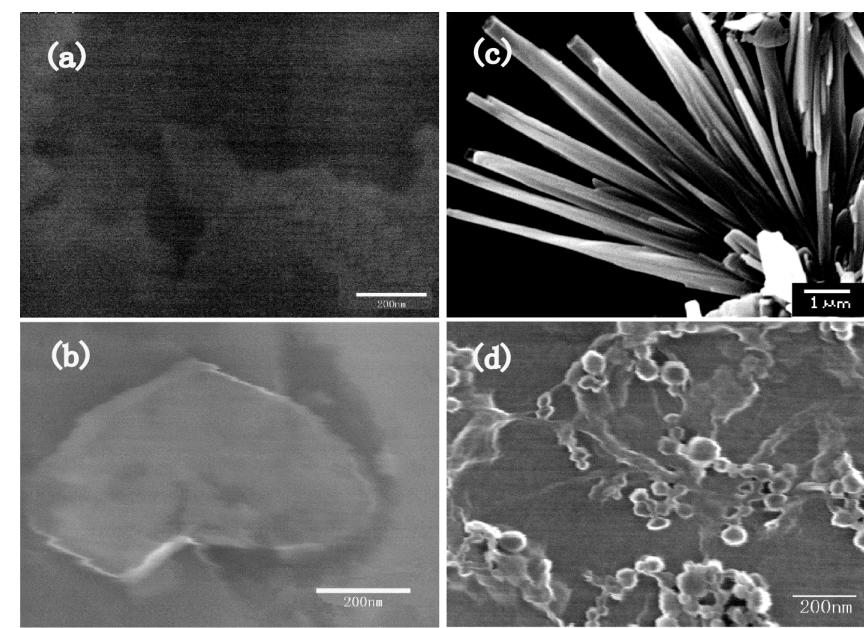

Fig. 1. SEM images of (a, b) graphene, (c) PANI and (d) PANI/graphene complex

The CV of bared, PANI and PANI/graphene complex modified GCE in $\mathrm{N}_{2}$-saturated PBS solution were shown in Fig. 2. As shown in this figure, the oxidation and reduction currents at the bare GCE were quite small. The electrochemical responses obtained at the $\mathrm{PANI} /$ graphene/GCE were much larger than that obtained at the PANI/GCE and bared GCE. The higher catalytic current and earlier onset potentials in the process of both oxidation and reduction at PANI/graphene/ GCE were observed. It showed that the electrical activity of electrode had been improved obviously after modificated by graphene/PANI composite. The electrochemical activity of PANI modified GCE in neutral was electrochemical inert, which was consistent with those reported ${ }^{20}$. In comparison with the CV curve of PANI modified GCE, it can be concluded

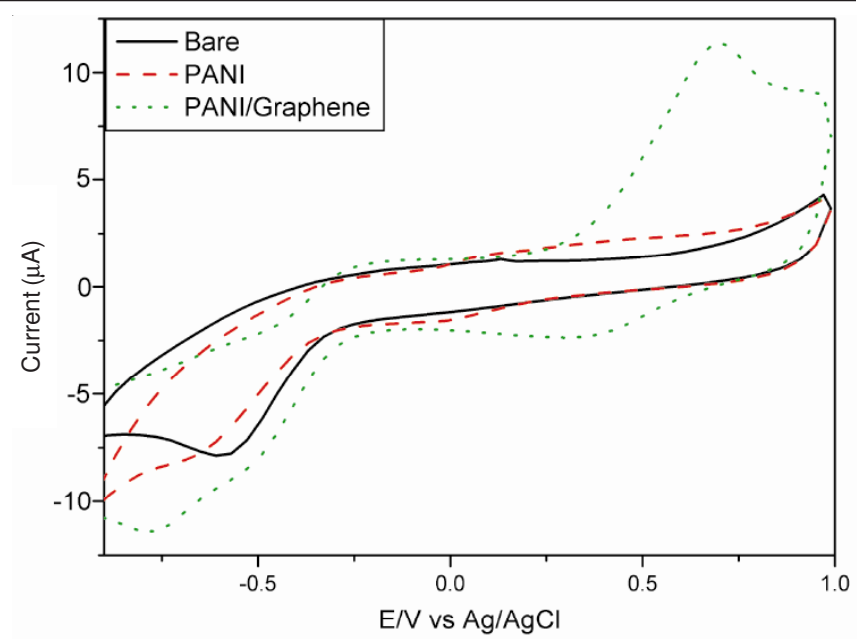

Fig. 2. Cyclic voltammograms of the bare, PANI and PANI/graphene complex modified GCE in PBS $(\mathrm{pH}=7.0)$ with the scan rate 50 $\mathrm{mV} \mathrm{s}^{-1}$

that the redox waves should be ascribed to the synergy of graphene and PANI on the electrocatalytic activity.

Based on the good electrocatalytical activity of PANI/ graphene/GCE to $\mathrm{H}_{2} \mathrm{O}_{2}$ and $\mathrm{O}_{2}$, the a glucose biosensor was further developed. The CVs of graphene/PANI/GOD modified GCE at various scan rates ranging from $10-300 \mathrm{mV} \mathrm{S}^{-1}$ were investigated (Fig. 3). The potentials and peak currents were dependent on the scan rate. In addition, both the anodic and cathodic peak currents were directly proportional to the square root of rates (Fig. 3). It indicated that the redox process of GOD in this composite film was a reversible diffusion controllable redox process.

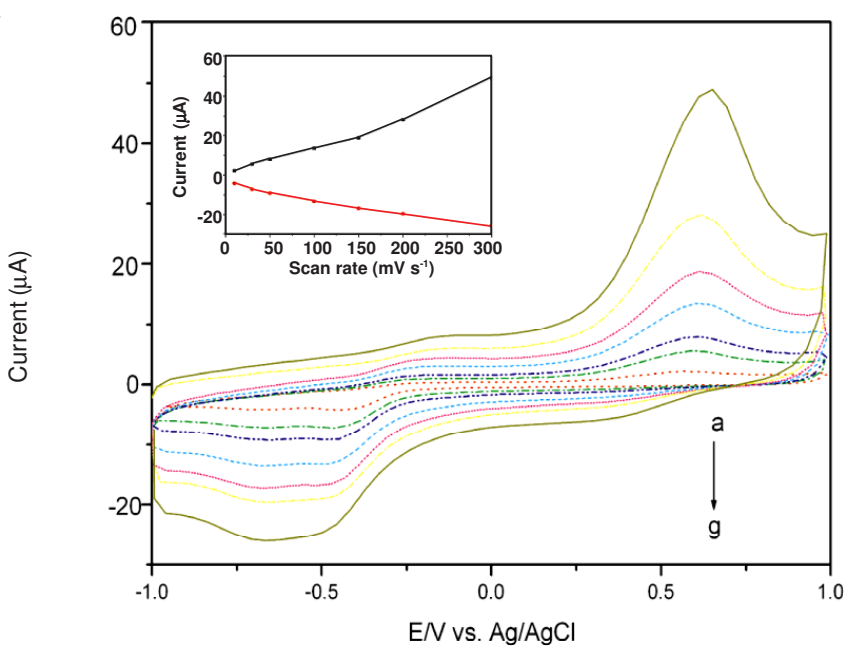

Fig. 3. CVs of PANI/graphene/GOD in $0.05 \mathrm{M} P B S(\mathrm{pH}=7.0)$ at various scan rates (a-h: 10, 30, 50, 100, 150, 200, $300 \mathrm{mV} \mathrm{s}^{-1}$ ). The inset showed the dependence of redox peak currents on the square root of scan rate

Fig. 4 showed the amperometric response of glucose biosensor to detect various concentrations of glucose PBS solution saturated with $\mathrm{O}_{2}$. The reduction current originating from reduction of $\mathrm{O}_{2}$ and $\mathrm{H}_{2} \mathrm{O}_{2}$ became smaller and smaller with the increase of glucose concentration. It resulted from the consumption of $\mathrm{O}_{2}$. Moreover, the calibration curve corresponding to amperometric response was linear against the 


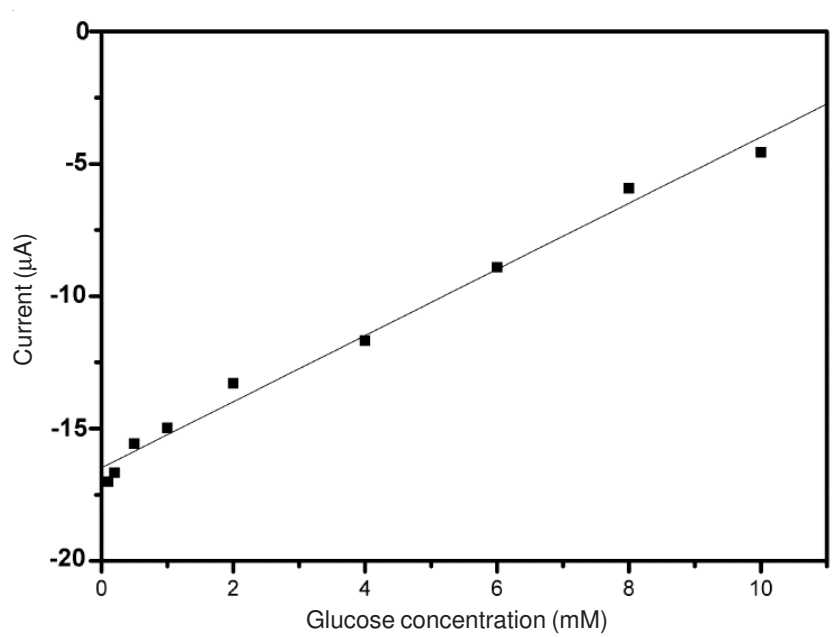

Fig. 4. Calibration curve of the PANI/graphene/GOD nanocomposite-based biosensor to detect various concentrations of glucose PBS solution saturated with $\mathrm{O} 2$. It was corresponding to amperometric responses at various concentrations of glucose with the scan rate $50 \mathrm{mV} \mathrm{s}^{-1}$

concentrations of glucose ranging from 0.1 to $10 \mathrm{mM}$ at -0.68 $\mathrm{V}(\mathrm{R}=0.994)$. As known, the blood glucose level of normal person is maintained between about 4 and $6 \mathrm{mM}$. So the linear glucose response from $0.1-10 \mathrm{mM}$ based on graphene/PANI/ GOD modified electrode was suited for the practical application in determining blood sugar concentration. Furthermore, it had good reproducibility and long-term stability. The relative standard deviation (RSD) of the current response to $6 \mathrm{mM}$ glucose was $3.2 \%$ for 10 successive measurements. The response current was increased by 3.5 and $4.9 \%$ of its initial response after 3 days and 1 week, respectively.

\section{Conclusion}

In summary, we presented a novel bionanocomposite film consisting of PANI/graphene/GOD for glucose detection. The glucose biosensor exhibited good sensitivity with a wide linear response from 0.1-10 mM. Such sensitivity was attributed to the synergy of graphene and PANI on the electrocatalytic activity. The glucose biosensor had good responses because of the large surface area and fast electron transfer of graphene and PANI. This work showed that the hybrid PANI/graphene/ GOD nanocomposite-based biosensor had great potential for clinical utility and home care for a rapid monitoring of glucose.

\section{ACKNOWLEDGEMENTS}

This work was supported by grants from the National Basic Research Program of China (973 Program), No. 2012CB933303, 2011CB707505, the National Science Foundation of China (No. 31000791, 41073060, 50874032, 21275153), the National Key Technology R\&D Program (No. 2012BAK08B05), and Science and Technology Commission of Shanghai Municipality (No. 11391901900, 11530700800, 11ZR1443900, $11 \mathrm{~nm} 0505800$ and 12441902600, 10391901600).

\section{REFERENCES}

1. R.C. Barry, Y. Lin, J. Wang, G. Liu and C.A. Timchalk, J. Expo. Sci. Environ. Epidemiol., 19, 1 (2009).

2. V. Velusamy, K. Arshak, O. Korostynska, K. Oliwa and C. Adley, Biotechnol. Adv., 28, 232 (2010).

3. T. Selvaraju, J. Dasa, S.W. Han and H. Yang, Biosens. Bioelectron., 23, 932 (2008).

4. D. Chung, K. Kim and S. Choi, Appl. Surf. Sci., 257, 9390 (2011).

5. B.J. Privett, J.H. Shin and M.H. Schoenfisch, Anal. Chem., 80, 4499 (2008).

6. N. Alexeyeva, J. Kozlova, V. Sammelselg, P. Ritslaid, H. Mändar and K. Tammeveski, Appl. Surf. Sci., 256, 3040 (2010).

7. A.K. Geim and K.S. Novoselov, Nat. Mater., 6, 183 (2007).

8. Z. Liu, Q. Liu, Y. Huang, Y. Ma, S. Yin, X. Zhang, W. Sun and Y. Chen, Adv. Mater., 20, 3924 (2008).

9. S. Guo and S. Dong, Chem. Soc. Rev., 40, 2644 (2011).

10. M. Zhou, Y. Zhai and S. Dong, Anal. Chem., 81, 5603 (2009).

11. Y. Shao, J. Wang and H. Wu, J. Liu, I.A. Aksay and Y. Lin, Electroanalysis, 22, 1027 (2010).

12. Y. Lin, K.A. Jenkins, A. Valdes-Garcia, J. P. Small, D.B. Farmer and Ph. Avouris, Nano Lett., 9, 422 (2009).

13. D. Wang, F. Li, J. Zhao, W. Ren, Z. Chen, J. Tan, Z. Wu, I. Gentle, G. Q. Lu and H. Cheng, ACS Nano, 3, 1745 (2009).

14. C.E. Banks, T.J. Davies, G.G. Wildgoose and R.G.. Compton, Chem. Commun., 7. 829 (2005).

15. Y. Wang, Y.M. Li, L.H. Tang, J. Lu and J.H. Li, Electrochem. Commun., 11, 889 ( 2009).

16. S. Alwarappan, A. Erdem, C. Liu and C.Z. Li, J. Phys. Chem. C, 113, 8853 (2009).

17. W.S. Hummers and R.E. Offeman, J. Am. Chem. Soc., 80, 1339 (1958).

18. J. Zhang, F. Zhang and H. Yang, Langmuir, 26, 6083 (2010).

19. J.X. Huang and R.B. Kaner, J. Am. Chem. Soc., 126, 851 (2004).

20. A.F. Diaz, J.A. Logan, J. Electroanal. Chem., 111, 111 (1980). 